AperTO - Archivio Istituzionale Open Access dell'Università di Torino

\title{
Disorders of higher cortical function
}

\section{This is the author's manuscript}

Original Citation:

Availability:

This version is available http://hdl.handle.net/2318/149970

since

Publisher:

Academic Press (ELSEVIER)

Terms of use:

Open Access

Anyone can freely access the full text of works made available as "Open Access". Works made available under a Creative Commons license can be used according to the terms and conditions of said license. Use of all other works requires consent of the right holder (author or publisher) if not exempted from copyright protection by the applicable law. 
AUTHOR QUERY FORM

\begin{tabular}{|c|c|c|}
\hline ELSEVIER & $\begin{array}{l}\text { Book: ZIGMOND-9780123982704 } \\
\text { Chapter: } 32\end{array}$ & $\begin{array}{l}\text { Please e-mail your responses and any corrections to: } \\
\text { E-mail: c.wortley@elsevier.com }\end{array}$ \\
\hline
\end{tabular}

Dear Author,

Any queries or remarks that have arisen during the processing of your manuscript are listed below and are highlighted by flags in the proof. (AU indicates author queries; ED indicates editor queries; and TS/TY indicates typesetter queries.) Please check your proof carefully and answer all AU queries. Mark all corrections and query answers at the appropriate place in the proof (e.g., by using on-screen annotations in the PDF file http://www.elsevier.com/book-authors/science-and-technology-book-publishing/ overview-of-the-publishing-process) or compile them in a separate list, and tick off below to indicate that you have answered the query.

Please return your input as instructed by the project manager.

\begin{tabular}{|l|l|}
\hline Location in Chapter & Query / remark \\
\hline & No Query \\
\hline
\end{tabular}




\title{
Disorders of Higher Cortical Function
}

\author{
Anna Berti*, $\dagger$, Francesca Garbarini*, Marco Neppi-Modona*, $\dagger$ \\ *Psychology Department, University of Turin, Turin, Italy; ${ }^{\dagger}$ Neuroscience Institute of Turin (NIT), University \\ of Turin, Turin, Italy
}

\begin{tabular}{|c|c|c|c|}
\hline \multirow{3}{*}{$\begin{array}{l}\text { Introduction: From Neuropsychology to Mental } \\
\text { Structure }\end{array}$} & \multicolumn{3}{|c|}{ O U T L I N E } \\
\hline & & Disorders of Visual Recognition: Agnosia & 8 \\
\hline & 1 & Object Agnosia & 8 \\
\hline Lanouaoe Disorders & & Visual Agnosia & 8 \\
\hline Language Disorders & $\begin{array}{l}3 \\
3\end{array}$ & Apperceptive Agnosia & 8 \\
\hline Aphasia: Definition and Treatments & $\begin{array}{l}3 \\
2\end{array}$ & Associative Agnosia & 9 \\
\hline Classical Aphasia & 3 & Tactile Agnosia & 9 \\
\hline Broca Aphasia & 3 & Acoustic Agnosia & 10 \\
\hline Wernicke Aphasia & 4 & Face Agnosia (Prosopagnosia) & 10 \\
\hline Conduction Aphasia & 4 & Apperceptive and Associative Prosopagnosia & 10 \\
\hline Global Aphasia & 4 & Unconscious Face Recognition & 10 \\
\hline Transcortical Aphasias & 4 & Unconscious Face Kecognition & 10 \\
\hline Anomic Aphasia & 4 & Disorders of Spatial Representation: Unilateral & \\
\hline Towards a More Complex Model of Language in the & & Neglect & 10 \\
\hline Human Brain & 4 & Clinical Manifestations & 10 \\
\hline Embodied Semantics & 5 & Clinicoanatomical Correlations & 11 \\
\hline Memory Disorders: Amnesia & 5 & Course of Unilateral Neglect & 11 \\
\hline & & Interpretation of the Syndrome & 11 \\
\hline Disorders of Movement Execution: Apraxia & 6 & Sensory Interpretations & 11 \\
\hline Ideomotor Apraxia & 6 & Attentional Interpretations & 11 \\
\hline Ideational Apraxia & 6 & Representational Interpretations & 12 \\
\hline Orofacial Apraxia & 7 & Implications for Cognitive Science & 12 \\
\hline Limb-Kinetic Apraxia & 7 & Conscious Awareness & 13 \\
\hline Constructional Apraxia & 7 & Blindsight: A Model for Visual Awareness & 13 \\
\hline Anatomy of Apraxia & 7 & Neglect: A Model for Spatial Awareness & 14 \\
\hline Interhemispheric Localization & 7 & Anosognosia for Hemiplegia: A Model for Motor & \\
\hline Intrahemispheric Localization & 7 & 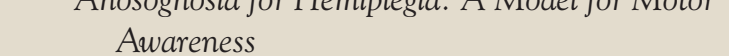 & 14 \\
\hline Neuropsychological Models of Apraxia & 7 & A Modular Model for Conscious Awareness & 15 \\
\hline Liepmann's Model & 7 & Future Directions & 16 \\
\hline Geschwind's Model & 8 & Future Directions & 10 \\
\hline $\begin{array}{l}\text { Heilman and Rothi's Model } \\
\text { Key Questions }\end{array}$ & $\begin{array}{l}0 \\
8\end{array}$ & References & 16 \\
\hline
\end{tabular}


s0010

\section{INTRODUCTION: FROM NEUROPSYCHOLOGY TO MENTAL STRUCTURE}

p0010 Focal brain lesions, that is, pathological events such as ischemic attack, tumors, and even infective diseases that affect relatively small parts of the cerebral cortex, can cause substantial but circumscribed impairments of either primary or cognitive nervous function, or both. The observed alterations depend on the areas and the cerebral circuits involved. If the lesion affects the primary motor areas, which are located in the frontal lobes of both hemispheres, and in particular in Brodmann area (BA) 4, and the axons that go from this cortical area to the spinal motoneurons, a paresis contralateral (most of the sensorimotor pathways are crossed) to the brain damage will be observed. Similarly, damage to primary sensory areas and pathways would cause contralateral sensory disturbances (e.g. anesthesia of contralateral limbs when sensory parietal cortices are damaged or blindness of one visual hemifield from damage to the primary visual areas in the occipital lobes). When the damage is localized outside primary motor and sensory cortices, complex cognitive deficits may be observed, involving what are called higher brain functions. In these latter cases the observed disorders may range from impairments of the cognitive analysis of input stimuli to impairments of the motor programming and selection of output signal (therefore, aspects that, although related to perception and movements, cannot be ascribed to the simple deficit in stimulus analysis or to the strength deficit related to the paralysis) or may involve functions such as language, memory, decisional processes, or domainspecific disturbance of conscious awareness (see below).

p0015 The discipline that studies the relationship between brain damage and higher cognitive functions is called neuropsychology. While its clinical goal is the description, diagnosis, and treatment of the disorders consequent upon the brain damage, the main experimental aim, as widely discussed in the seminal book by Tim Shallice, From Neuropsychology to Mental Structure, ${ }^{1}$ is to draw inferences from the pathological conditions to normal functions, on the assumption that if the lesion to a particular brain area has provoked a specific deficit then that part of the brain is involved, underpins, or is necessary to sustain the normal function. Scientific neuropsychology dates back to the second half of the nineteenth century, when the observation that there was a strict relation between focal brain damage and disturbances of higher mental functions became a matter of debate among neurologists, who started to systematically study and report single cases of patients with domainspecific cognitive impairment to unveil the neural bases of cognition. The observation that cognitive impairment could derive from specific brain damage gave rise to many studies that not only described the relation between the symptoms and the lesion but also proposed real "models" for the altered function. Indeed, Broca in $1861^{2}$ not only suggested a relationship between the presence of a brain lesion and a language problem, but indicated in the third frontal circonvolution the exact locus of linguistic functions, therefore establishing a precise anatomoclinical correlation.

The first neuropsychologists, on the bases of their p0020 observations, proposed simple models of cerebral functions in the form of diagrams in which gray matter structures, underlying higher cognitive functions, and white matter pathways, connecting different centers, were represented. These models could explain the disorders already observed and also tried to predict new syndromes on the basis of the acquired knowledge. The great merit of the "diagrammists" was that they realized that brain injuries can elucidate the organization of the cognitive systems. Although observations similar to those reported by Broca had been made previously, the scientific community was not ready to accept them. The scientific discoveries in neurology and neurophysiology, which identified in specific brain areas specialized regions for the control of sensorimotor functions, also created the cultural conditions for considering a cause-effect relationship between injury and impaired function plausible for cognitive disorders, thus initiating a localization-association approach for the study of higher brain functions. Although the acceptance of the relationship between brain injury and altered behavior paved the way for the study of the neurobiological bases of cognitive functions, these models had some limitations, mainly related to the lack of solid psychological theories, the idiosyncratic choice of the patients to be studied, and the lack of methodological constraints and quantitative analysis. Therefore, the neuropsychological models were adjusted and adapted over the course of thetwentiethcentury depending on the theoretical paradigms that were dominant in the neuroscientific and psychological fields.

First, the classical method used by neuropsychology, p0025 the study of clinical cases, underwent some changes owing to the modified scientific conditions. The development of anatomical knowledge, on the one hand, and the need to apply a quantitative approach to the study of patients, which would allow a greater objectivity for neurological observations and a less idiosyncratic choice of the patients to be studied, on the other hand, imposed, in the second half of the twentieth century, the transition from the study of individual isolated cases to the study of groups of patients, who were selected according to strict criteria established a priori. For instance, patients could be selected on the basis of the symptom one wanted to study or according to the lesion site. Control groups of neurologically intact subjects were also considered as the normal reference system. 
p0030 At the same time, the study of neuropsychological disorders began to be standardized through the use of validated tests, which permitted the gathering of data suitable for statistical evaluation. These advances led to the acquisition of reproducible and fundamental knowledge on various aspects of human cognition, mainly related to the differences in intrahemispheric and interhemispheric neurofunction. In the second half of the twentieth century, the introduction of the cognitive psychology approach and the model of human information processing, which proposed a multicomponent structure of the cognitive system, provided a useful theoretical framework for the study of the injured brain. Indeed, if it is plausible to assume the existence of functionally segregated cognitive centers, then it is possible that a center could be selectively damaged by a brain lesion, causing a very specific and isolated disorder of cognition. As Smith Churchland puts it: "So long as the brain functions normally, the inadequacies of common-sense framework can be hidden from view, but with a damaged brain the inadequacies of theory are unmasked". ${ }^{3}$

p0035 The general model of the functioning of the cognitive system was not very different from the diagrammists' models insofar as different brain centers were supposed to give rise to separated and anatomically segregated cognitive functions, connected by interhemispheric and intrahemispheric pathways, and the single case method was again considered the ideal way to unveil the structure of the cognitive system. However, in the modern version of the classical associations, clinical cases are evaluated with both sophisticated methodological and statistical criteria and detailed in vivo neuroanatomical investigations. It is worth noting that studies in patients with brain lesions, obtained with the methods of the modern neuropsychological research, have often disclosed the limits of the anatomoclinical inferences related to the idea that brain function is localized in a single area of cortical tissue. Often similar disorders can be observed in the presence of damage in different brain structures, which suggests that a given function is not localized in one single area of the brain, but that the damaged region is part of a circuit or system constituted by several cortical-subcortical centers. The idea that a function is distributed in the brain does not mean that all brain areas participate equally in that function, but that the function depends on the cooperation of specific, but different, parts of the brain. Therefore, although adjusted by the newly acquired knowledge on cerebral organization, the principle that focal damage to the brain can cause circumscribed and specific impairment of cognitive function still holds.

p0040 Lesion studies have significantly contributed to the definition and clarification of the cognitive prerogative of the human mind, falsifying the ultracognitive view that mental operations, made possible by our information processing systems, do not depend on the structural characteristics and organization of the biological substrate with which we are endowed.

\section{LANGUAGE DISORDERS}

As already mentioned, the birth of modern neuropsy- p0045 chology is usually identified as Paul Broca's presentation at the Société Anatomique, in 1861, of his study on the anatomical localization of the articulated language in the inferior frontal gyrus. ${ }^{2}$ In Broca's pioneering findings, the tight link between cognitive functions and neuroanatomical structures became evident: if a specific structure (the "Broca area") is damaged, a specific function (speech production) is lost. This section will focus on the neuropsychological study of language deficits, from the "classical" aphasias to a more complex model of the linguistic functions inside the human brain. A new research field, aiming to explain "normal" linguistic processing within the theoretical context of the embodied semantic, is also investigated.

\section{Aphasia: Definition and Treatments}

Aphasia is a disturbance of the production and/or the p0050 comprehension of language caused by selective damage in specific brain areas, usually located in the left hemisphere (which is dominant for language in both righthanded and left-handed people). In aphasia, multiple aspects of language can be compromised, including the syntax (the grammatical structure of sentences), the lexicon (the collection of words that denote meanings), and the morphology (the combination of phonemes, single speech sounds, into morphemes, the smallest meaningful units of a word). There are different profiles of aphasia, depending on which of these linguistic aspects is most compromised in a specific patient. Most cases of aphasia are caused by trauma or stroke, cerebral tumors, or degenerative dementias. The correct diagnosis and effective treatment of aphasia have a great clinical importance because this deficit has a devastating impact on the patient's quality of life. Clinical interventions for aphasia aim to activate dysfunctional brain networks supporting linguistic processing and communicative intent. These interventions generally fall into three broad categories: speech-language rehabilitation treatments; pharmaceutical treatments; and direct brain-stimulation therapies, such as transcranial magnetic stimulation and transcranial direct current stimulation. After a brain injury there is a spontaneous recovery period, but there is converging evidence that language recovery may be enhanced by a program of rehabilitation therapy. 


\section{s0025 Classical Aphasia}

p0055 Historically, on the basis of both clinical and postmortem anatomical observations, language has been localized in two major brain areas: Broca anterior frontal area (Brodmann area, BA44) for speech production and Wernicke posterior temporal area (BA22) for speech comprehension. The first functional model of language, proposed by Lichtheim in $1885,{ }^{4}$ was an attempt to explain linguistic processing within this left temporofrontal network, giving an account of a wide range of known aphasic symptoms. The novelty of Lichtheim's approach was to define language syndromes in terms of damage to the components of the model, in the same way as neuropsychologists nowadays do with the large spectrum of sensory, motor, and cognitive deficits affecting brain-damaged patients.

p0060 In the classical view of aphasia the following syndromes were described. ${ }^{5}$

\section{s0030 Broca Aphasia}

p0065 There are two variants of Broca aphasia. In classical Broca aphasia, lesions actually involve not only Broca area (BA44 and 45) but also the surrounding frontal regions (BA8, 9, 10, and 46) and the underlying white matter and basal ganglia. Patients have a dramatic loss of speech fluency and a specific form of agrammatism, characterized by the inability to organize words and sentences according to grammatical rules. Moreover, the patients' capacity to repeat sentences is compromised, as well as their ability to assemble phonemes correctly (they often show phonemic and phonetic paraphasias). When lesions are restricted to the Broca area, a milder and more transient form is observed. Traditionally, from Broca's original description, ${ }^{2}$ Broca aphasia is considered a deficit of speech production, but some of the difficulties noted in language production have also been found to occur in language comprehension, in particular when the grammatical structure is complex as, for instance, in passive sentences.

\section{s0035 Wernicke Aphasia}

p0070 Wernicke aphasia is caused by damage to the posterior sector of the left auditory association cortex (BA22), often involving other surrounding areas (BA37, 39, and 40 ) and the underlying white matter. In patients with Wernicke aphasia, speech is fluent (effortless, melodic, and produced at normal rates) but the content is often unintelligible because of frequent errors in phoneme and word choice (patients often show phonemic and semantic paraphasias and neologisms). Patients with Wernicke aphasia have difficulties in comprehending sentences uttered by other people, showing a deficit at a semantic-lexical level. The deficit of comprehension can be extended to the written language.

\section{Conduction Aphasia}

s0040

Conduction aphasia shares with Broca and Wernicke p0075 aphasias the inability to repeat sentences, a defective assembly of phonemes, and an impaired naming ability, but it differs from them in the relatively preserved speech production and auditory comprehension. In classical descriptions (Wernicke's model ${ }^{6}$ and Lichtheim's model $^{4}$ ), conduction aphasia was uniquely ascribed to lesions of the arcuate fasciculus, a white matter pathway connecting the Wernicke and Broca areas. It is now known that, although the subcortical projections linking temporal, parietal, and frontal cortices are often damaged in patients affected by this kind of language problem there is no evidence that a pure white matter disconnection can cause conduction aphasia. In addition, the involvement of the left inferior parietal lobe (BA40), the left primary auditory cortices (BA41 and 42), and the insula seems to be necessary to cause the symptoms.

\section{Global Aphasia}

Patients with global aphasia have almost completely p0080 lost the ability to comprehend language and formulate speech, combining the features of both Broca and Wernicke aphasia. Global aphasia is usually caused by large anteroposterior damage, widely involving the language regions, the basal ganglia region, and the insula.

\section{Transcortical Aphasias}

These types of aphasia can be distinguished from all p0085 others by the fact that the ability to repeat sentences is normal. The motor variant usually occurs after left frontal lesions above and in front of the Broca area. The sensory variant is caused by lesions in temporal or parietal cortices, in the vicinity of the Wernicke area.

\section{Anomic Aphasia}

Damage to the left anterior temporal cortices (BA20, p0090 21, and 38) severely impairs the ability to retrieve words, but is not accompanied by any grammatical, phonemic, or phonetic difficulty, causing a "pure" naming deficit. More specifically, when the damage is confined to the left temporal pole (BA38) patients have a deficit in the ability to retrieve proper nouns of places and people; when the lesion involves BA20 and 21 the defect encompasses the ability to retrieve both proper and common nouns.

\section{Towards a More Complex Model of Language in the Human Brain}

Although the anatomoclinical correlations between p0095 the different types of aphasia symptom and the damage to specific brain areas were thoroughly confirmed in the twentieth century by a large number of neuropsychological and neurostructural studies, in recent decades 
the complex networks underlying language have been explored using innovative techniques such as functional magnetic resonance imaging (fMRI) and diffusion tensor imaging. This approach led to the development of a more dynamic, connectionist approach to the study of the anatomical correlates of aphasias. On the one hand, areas originally thought to be specialized for language have been shown to be also involved in cognitive and perceptual functions not directly related to language; on the other hand, it has been demonstrated that language does not exclusively rely on the Wernicke-Broca language network. ${ }^{7}$ Furthermore, recent neuropsychological studies on the consequences of acquired brain lesions in children and adults have suggested that the outcomes of aphasias have a greater variability than predicted by the classical models, depending on the patient's age, the etiology, site, and size of the lesion, and the initial severity of the impairment. ${ }^{8}$ Whereas sudden brain lesions affecting specialized areas often result in severe aphasia, the clinical pattern is different in the case of slowly growing lesions such as low-grade gliomas. Slow tumor evolution allows for compensatory mechanisms to develop through the recruitment of intrahemispheric and interhemispheric neuronal networks (i.e. perilesional and/or contralateral homologous brain regions). As suggested by the case study of a "patient speaking without Broca's area", 7 in which the left inferior frontal gyrus resection (including Broca area), due to tumor infiltration, did not lead to severe language impairments, the efficiency of brain plasticity can, in some instances, compensate for the anatomical specialization of linguistic functions.

\section{s0065 Embodied Semantics}

p0100 Neuropsychological research on language functions is not confined to the study of aphasia, but also includes the study of "normal" linguistic processing in healthy subjects. An innovative and fecund research paradigm has developed, which aims to explain linguistic functions within the theoretical framework of embodied cognition. ${ }^{9,10}$ From this perspective, not only perceptual representation but also high-level cognitive processes, such as concept formation and language, are essentially based on motor programs. Contrary to the classical approach in cognitive science, in which concepts are viewed as amodal and arbitrary symbols, the embodied hypothesis argues that concepts must be grounded on sensorimotor experiences to be meaningful. In line with this view, neuroimaging studies have shown a somatotopic pattern of activation along cortical motor areas for the observation of actions involving different body parts, as well as for action-related language comprehension (see Aziz Zadeh and Damasio ${ }^{11}$ for a review). For example, the concept of "grasping" would be represented in sensorimotor areas that code grasping actions; the concept of "kicking" would be represented by sensorimotor areas that control kicking actions; and so forth. The signals related to the common goal for a variety of specific actions (i.e. grasping with the mouth or with the hand or toes, along with related affordances) would also converge on a particular group of neurons, thus providing a more general representation of "grasping". This would be the neurobiological substrate of generalized conceptual representations, which, in turn, could be related to language description. ${ }^{11}$ This theory has been extended by Lakoff and colleagues to include metaphors. Thus, the phrase "kick off the year" would also involve the motor representations related to kicking, just as the phrase "grasp the explanation" would involve motor representations related to the control of the hand. ${ }^{12}$ In a future perspective, exploring the link between these conceptual representations and metaphorical language will be especially important, as it has been proposed that much of abstract thinking is performed metaphorically.12

\section{MEMORY DISORDERS: AMNESIA}

Amnesia, a profound disorder of memory functions, p0105 is a clear example of how we can propose a hypothesis about the structure of a cognitive process and make inferences from the combination of association and dissociation of symptoms present in a particular kind of neuropsychological syndrome. Since the work of Hebb, ${ }^{13}$ the idea that memory is equally distributed throughout different brain regions, being intimately related to perceptual and intellectual functions, ${ }^{14}$ was strongly challenged by those scientists who believed that, although memory processes are indeed distributed in different cortical and subcortical structures, specific aspects are processed by different areas and relatively independently from other cognitive functions. This latter position was dramatically confirmed by the study of patients who, immediately after selective brain surgery for the treatment of intractable epilepsy, showed dramatic, specific, and dissociated memory impairments. The most enlightening observations were made by Brenda Milner, Susan Corkin, and their co-workers ${ }^{15-17}$ on patient HM. This patient, after bilateral resection of the medial temporal lobes, was left with a complex syndrome characterized by severe memory impairments that affected some memory capacities but not others, and by a considerable sparing of other cognitive functions. First of all, it was noted that he had a severe anterograde amnesia, with which he could not acquire new memories, dating from the day of his brain damage. Moreover, he could not retrieve some of the memories that he acquired before surgery (retrograde amnesia), although access to facts and events from times remote from surgery were still possible. This latter finding implies that medial temporal lobe 
structures are not the final locus for storing old memories. After learning, memories that initially require the integrity of medial temporal lobe structures must be reorganized and stored somewhere else in the brain to become independent from these structures. According to many authors, once a memory has been fully consolidated, its storage and reactivation may depend on processes and structures located in neocortex. ${ }^{18}$ The extent of retrograde amnesia may be taken as an index of how long the consolidation process lasts.

p0110 Despite his severe memory problems, HM's other cognitive and perceptual capacities were intact. For instance, his intelligence quotient (IQ) was higher after compared with before surgery and no main perceptual problems were detected. The memory disorders affected both verbal and non-verbal tasks, as well as stimuli presented in all sensory modalities. From these observations the inference was made that memory functions can be kept separated from other cognitive functions and from more primary perceptual and sensory processes. ${ }^{18}$ It is also worth noting that the impairments described above involved what are normally indicated as long-term memory capacities. However, short-term memory was intact in HM. That means that he could repeat a sequence of digits immediately after their presentation and that he could follow a conversation providing that it did not become too long and based on data that were presented before the talk. ${ }^{19}$ Therefore, the dissociation between long and short memory processes was another fundamental finding from the study of HM.

p0115 As already mentioned, within long-term memory processes, anterograde amnesia refers to the impossibility of learning new facts and HM was severely impaired in this domain. However, and somehow astonishingly considering his otherwise dramatic memory impairment, HM showed intact capacities of acquiring new motor and perceptual skills ${ }^{20-22}$ (procedural memory), clearly demonstrating that amnesia associated with hippocampal damage affects what are indicated as declarative memory processes (the ability to recall facts and events related to specific personal experience, episodic memory), leaving intact other memory operations related to the functioning of separated brain structures and systems. ${ }^{23}$ It is important to note that HM's profound memory disorder resulted from brain damage that was not confined to the hippocampus but also involved the amygdala and the adjacent parahippocamapal gyrus. Another patient, RB, who had a lesion limited to the hippocampus following an ischemic attack, had a similar, but less severe memory disorder. ${ }^{24}$ Taken together, these results indicate that damage to the hippocampus is sufficient to cause a clinically significant amnesic disorder and that additional damage to adjacent structures aggravates the symptoms.
The study of HM revealed the most important aspects p0120 of how memory is structured and organized in the brain. As Squire and Wixted wrote in 2011: "These findings established the fundamental principle that memory is a distinct cerebral function, separable from other perceptual and cognitive abilities, and also identified the medial aspect of the temporal lobe as important for memory. The early descriptions of HM can be said to have inaugurated the modern era of memory research, and the findings from HM enormously influenced the direction of subsequent work".

It is worth remembering that there are, unfortunately, p0125 other pathological conditions that can induce severe amnesic disorders. Alcohol abuse, leading to thiamine deficit and to damage to diencephalic brain structures, including the mammillary body, can be the cause of Korsakoff syndrome, where a severe anterograde deficit is always observed (for a review see Fama et al. ${ }^{25}$ ). Infections affecting the brain, such as herpes simplex virus encephalitis, can cause disastrous amnesia that may extend beyond episodic problems and involve semantic knowledge.

\section{DISORDERS OF MOVEMENT EXECUTION: APRAXIA}

Apraxia has been defined as the inability to carry out p0130 learned, skilled motor acts despite preserved motor and sensory functions, coordination, and comprehension. The major types are described in this section.

\section{Ideomotor Apraxia}

Ideomotor apraxia (IMA) is the impaired ability to p0135 perform a skilled gesture with a limb upon verbal command and/or by imitation. It can be shown for both meaningful motor acts that do not imply objects and gestures that imply object use. In this latter case, patients may not be able to perform the pantomime (i.e. to show how to use an object without actually manipulating it) or may not be able to use the actual object. Many authors also consider the inability to imitate meaningless gestures as apraxia. Patients with IMA show errors in the temporal and spatial sequencing of movements, in their amplitude and configuration, and in limb position in space.

Various dissociations have been described. For instance, p0140 a voluntary-automatic dichotomy has been described according to which patients seem to be able to perform in their daily life activities the same acts that they are unable to perform when requested by the examiner. Differences in the performance related to the type of gesture to be produced have also been described. For instance, there may be differences in the performance between actions implying use of an object (transitive actions) and actions not 
implying use of an object (intransitive actions, e.g. waving goodbye). Finally, apraxia is often more severe for meaningless actions than for meaningful actions, although the opposite dissociation has also been described. Given these dissociations, it is essential that the diagnosis of IMA include a wide variety of tasks.

\section{s0085 Ideational Apraxia}

p0145 Patients with ideational apraxia (IA) have difficulties in executing a sequence of actions when performing a complex multistage task (e.g. making coffee). Some authors also distinguish between IA and conceptual apraxia, identifying with this latter condition an impairment of object or action knowledge. Patients with conceptual apraxia may misuse objects, can be impaired in matching objects with the corresponding actions, or may be unable to judge whether an action is correctly or ill formed. Patients with IA or conceptual apraxia tend to be seriously disabled in their everyday life. IA and IMA can be doubly dissociated.

\section{s0090 Orofacial Apraxia}

p0150 Orofacial apraxia is an impairment in the execution of skilled movements involving the face, mouth, tongue, larynx, and pharynx (e.g. blowing a kiss or whistling) when requested by the examiner. Similarly to IMA, automatic movements of the same muscles are often preserved. Orofacial and limb apraxias often coexist but can be dissociated.

\section{s0095 Limb-Kinetic Apraxia}

p0155 The term limb-kinetic apraxia describes inaccurate or clumsy distal movements of the limbs contralateral to the lesioned hemisphere. Limb-kinetic apraxia differs from classical ideomotor apraxia because it tends to be independent of modality (e.g. verbal command versus imitation) and there is typically no voluntary-automatic dissociation.

\section{s0100 Constructional Apraxia}

p0160 Constructional apraxia is a particular type of apraxia where patients are unable to spontaneously draw objects, copy figures and build blocks or patterns with sticks following damage to the left or right hemisphere. Hence, constructional apraxia seems to reflect the loss of the ability to integrate perceptual, categorical, and coordinate spatial relations with the motor actions necessary to complete a constructive task. constructional apraxia can be dissociated from the other types of apraxia.

\section{Anatomy of Apraxia}

\section{Interhemispheric Localization}

The two major forms of apraxia (IMA and IA) are more p0165 frequent following left hemisphere lesions. Nevertheless, in a few instances apraxia has also been described after lesions of the right hemisphere, suggesting that right hemisphere structures can also support skilled movements to a certain extent. Specifically, right hemisphere "praxic" structures can be recruited following injuries to the left hemisphere and also as a result of training and execution of highly practiced familiar actions.

\section{Intrahemispheric Localization}

IMA and IA have been associated with lesions to the p0170 parietal and frontal cortices of the left hemisphere, as well as with white matter connections between these areas. fMRI studies during gesture execution in healthy subjects have confirmed the involvement of left parietal and frontal regions, including the dorsolateral frontal and intraparietal cortex. A particular form of IMA can be seen after callosal lesions; in this case, it only involves the left hand, being the consequence of the disconnection of the right hemisphere premotor and motor areas from the left hemisphere praxis centers.

The basal ganglia also play an important role in praxis p0175 via bilateral connections with frontal and parietal areas.

Finally, it is important to consider the relationship p0180 between limb apraxia and aphasia. Apraxia and aphasia frequently coexist following left hemisphere damage, raising the question of whether there is a common feature underlying both disturbances (some authors point to an asymbolic problem) or whether linguistic dysfunction is responsible for apraxia because patients do not understand the examiner's requests. However, dissociations between aphasia and apraxia have been reported, thereby falsifying both hypotheses. Moreover, aphasia cannot account for apraxia in cases of gesture imitation. So, rather than there being a causal relationship between aphasia and apraxia, these two symptoms are likely to be associated in the same patient owing to lesions affecting adjacent neural substrates for language and gestures.

\section{Neuropsychological Models of Apraxia}

Different models to understand the neurocogni- p0185 tive mechanisms underlying apraxic disorders have been proposed. Here, the most influential ones will be reviewed.

\section{Liepmann's Model}

In 1920, Hugo Liepmann proposed a disconnection p0190 model of praxic disorders according to which the representation of an action (space-time plan) is stored in the left parietal lobe. ${ }^{26}$ In order to execute the action with 
the right hand, the space-time plan is retrieved and then reaches the primary motor areas through the left prefrontal cortex. Left-hand actions (ultimately controlled by the right hemisphere) are possible because the action plan reaches right premotor and motor areas through the corpus callosum. In ideomotor apraxia action representations and limb kinetics are intact, whereas frontoparietal connections are disrupted, causing an inability to execute normal actions. Ideational apraxia would result from direct lesion to the action representation area and limb-kinetic apraxia from disruption of "kinestheticinnervatory engrams" in the left frontal lobe.

\section{s0130 Geschwind's Model}

p0195 Norman Geschwind proposed a disconnection model of apraxia routed on Liepmann's model, according to which the verbal command for the action of the right hand is comprehended in the Wernicke area and is transferred to the ipsilateral premotor and motor areas via the superior longitudinal fasciculus. ${ }^{27}$ A lesion to this pathway would spare gesture comprehension but compromise action performance elicited by verbal command. To explain failure of action imitation and object use, Geschwind proposed that visual association and premotor areas are connected through the same pathways running through language and motor areas, but this notion remains controversial.

\section{s0135 Heilman and Rothi's Model}

p0200 Heilman and Rothi proposed an alternative representational model of apraxia in which left anterior premotor-motor regions are responsible for gesture production and left posterior parietal regions store the representation of learned movements and are responsible for gesture comprehension and discrimination..$^{28}$ Lesions to anterior regions would cause ideomotor apraxia, and lesions to posterior regions ideational apraxia as well as difficulties in movement production. Some neuropsychological data have supported this model.

p0205 To account for modality-specific dissociations in praxic disorders, in subsequent versions of their model Heilman and Rothi proposed separate processing routes for auditory and visual inputs, conveying information in a specific "action semantic system", dissociable from other semantics, which would activate an "action reception lexicon" connected with an "action production lexicon". ${ }^{29}$ A separate "non-lexical route", which bypasses the action semantic system, would be responsible for the ability to imitate novel and meaningless gestures.

\section{s0140 Key Questions}

p0210 The discovery that particular neuronal populations (mirror neurons) are active both when an action is produced and when an individual observes that same action ${ }^{30}$ poses a challenge for praxis models that hypothesize separate structures for action production and action recognition/comprehension. In particular, if the same representations subserve both action execution and recognition, the question arises of whether the perception/comprehension of a movement is constrained by its executional knowledge. The complexity of the praxis system suggests that it should be better studied through a multidisciplinary approach encompassing knowledge from lesion studies, fMRI data from healthy individuals, neurophysiological data from animal studies, and psychophysical and computational models of complex action execution (for reviews see references $\left.{ }^{31-33}\right]$. This could help to reach a more detailed mapping of large-scale neuronal networks underlying praxis, the assessment of alterations in these networks following injury, and their relationship with behavioral dysfunctions. A better understanding of these issues will lead to the development of more efficacious therapeutic and rehabilitative interventions.

\section{DISORDERS OF VISUAL RECOGNITION: AGNOSIA}

The neuropsychological disorder known as agnosia p0215 refers to the impairment of stimulus recognition in one modality in the absence of perceptual deficits, memory problems, and general intellectual impairment. This disorder is intriguing both scientifically and clinically and its study has contributed to shedding light on how the normal visual system functions. When the impaired recognition relates to objects in general, the condition is called object agnosia; when the unrecognized visual stimulus is a face, it is called prosopagnosia. An overview of the major types of agnosia is presented here.

\section{Object Agnosia}

In object agnosia, patients do not recognize objects in p0220 one specific input modality (visual, tactile, or auditory), whereas the same objects can be promptly recognized when presented through a different input channel. The perceptual nature of the disorder is testified by the fact that it cannot be ascribed to the co-occurrence of sensory elementary deficits, memory problems, naming difficulties (aphasia), and general intellectual impairment (patients are well aware of their predicament).

\section{Visual Agnosia}

Although it is a relatively rare neurological symptom, p0225 with some 100 cases published between 1890 and 1990, its study has greatly contributed to the understanding of how the process of visual recognition is organized in the human brain. There is no standard taxonomy of visual 
agnosias, but most neuropsychologists agree with Lissauer's original distinction between apperceptive and associative types, ${ }^{34}$ depending on the lower or higher processing stage of visual information affected by the brain lesion. Because this account has continued to be used in the neuropsychological literature to the present day, it is used here as a general framework.

\section{s0160 Apperceptive Agnosia}

p0230 Apperceptive agnosia is evident when patients are unable to recognize objects because they cannot see them properly, in the absence of elementary visual deficits. It is thought to arise from a breakdown at relatively early stages of visual processing, where the elementary features of the stimulus are analyzed. Object recognition through verbal description by the examiner is, instead, preserved. In apperceptive agnosia, shape perception is abnormal in such a way that patients cannot recognize or copy pictures, letters, or even simple geometric shapes. In most cases of apperceptive agnosia, the brain damage is diffuse, often caused by carbon monoxide poisoning. In the rare cases with circumscribed brain lesions, damage primarily affected the ventral occipitotemporal cortex bilaterally.

According to a widely accepted interpretation, apperceptive agnosia can be considered a deficit of shape perception resulting from defective perceptual grouping of an object's local features into a global percept. However, clinical findings show that apperceptive agnosia covers a wide spectrum of disorders, some of which fall in between apperceptive and associative agnosia. Hence, two neuropsychologists, Riddoch and Humphreys, ${ }^{35}$ proposed to differentiate between distinct subtypes of apperceptive agnosia, each corresponding to a defective processing stage along the hierarchically organized stream of visual information processing leading to conscious object perception (according to Marr's computational model of vision ${ }^{36}$ ). These subtypes are shape agnosia and integrative agnosia, which are closer to the apperceptive type; and transformational agnosia and agnosia due to impairment of internal object representation, which are closer to the associative type. ${ }^{35}$

u0010 - Shape agnosia results from a deficit of the initial processing stage of visual recognition and consists of the inability to organize the sensory input into a unified percept. Patients complain of blurred or unclear vision and are unable to discriminate stimulus boundaries from the background or other contiguous or overlapping shapes, as well as the orientation and size of the input.

u0015 - Integrative agnosia consists of the inability to integrate single object features into a global shape, in the presence of the ability to identify single object details. This deficit is more severe when the object shape is defined by high-frequency details or when overlapping figures must be identified, but is reduced when silhouettes of objects with reduced internal details are used for discrimination.

- Transformational agnosia is a deficit of perceptual categorization (first described in 1982 by Warrington ${ }^{37}$ ), which occurs when patients can recognize objects presented in a canonical view, but fail when they are presented in non-canonical views. What is lost is the ability to manipulate the mental representation of the object and to match it with its perceptual image.

- Agnosia due to impairment of internal representations of objects occurs when a structural description of the object is formed normally but its internal representation stored in presemantic memory cannot be accessed through a given route to match on-line descriptions encoded by the visual system.

\section{Associative Agnosia}

This type of agnosia occurs when patients can form p0260 a structural description of the visual object (object copy is preserved), yet are unable to recognize it. Associative agnosic patients cannot identify objects even by nonverbal means (e.g. by pantomiming their use or grouping together dissimilar objects from the same semantic category); however, recognition is preserved in the tactile modality (by touching the object) or from a spoken definition. Intrahemispheric location of the lesion is generally occipitotemporal, either unilateral (with a prevalence of left hemispheric lesions) or bilateral.

Associative agnosia has been explained as a deficit p0265 of the activation of the semantic associations related to the visual percept: patients, despite being able to form a normal visual representation of the stimulus, are unable to access the knowledge related to it and therefore recognition is prevented. Therefore, patients fail in semantic categorization and association tasks, as well as in the description of the semantic attributes of an object. In visual naming tasks, errors tend to be semantic (e.g. "knife" for "fork"), sometimes with the production of the superordinate ("flower" for "daisy"), although errors totally unrelated to the stimulus (e.g. "horse" for "chair") can be observed. Miming the use of a visually presented object is also impaired, whereas the task is carried out correctly if a verbal description of the object is offered. According to one view, associative agnosia is the consequence of a disconnection between visual areas and other brain centers responsible for language or memory. This hypothesis, however, does not account for the inability of agnosic patients to convey information non-verbally and to access old knowledge through vision. Another possibility is that semantic knowledge cannot be accessed through the visual modality because the lesion has damaged the 
connection between the areas that process the stimulus and semantic memory (semantic access agnosia). A different interpretation assumes that stored visual memory representations have been partially or totally damaged so that the newly formed visual percepts cannot be matched against any stored knowledge and, therefore, recognition is impossible. In this case, however, the disorder should be observed in all modalities and, therefore, cannot be considered a pure perceptual disorder but instead becomes a true disorder of semantic memory.

\section{s0170 Tactile Agnosia}

p0270 Tactile agnosia is the inability to recognize objects through touch, in the absence of elementary sensory deficits. Recognition in other modalities is preserved. The locus of the lesion involves the posterior-inferior portion of the parietal lobe and can be unilateral or bilateral. The deficit has been rarely studied and the interpretations are similar to those offered for visual agnosia.

\section{s0175 Acoustic Agnosia}

p0275 Acoustic agnosia (or aphasia) is the inability to name an object through sound (e.g. failure to name a bunch of keys given the sound the keys make when shaken) with preserved ability to recognize objects in other sensory domains.

\section{s0180 Face Agnosia (Prosopagnosia)}

p0280 The term prosopagnosia refers to the inability to recognize familiar faces. The deficit is confined to the identification of physiognomic traits, as shown by the fact that identification is preserved through non-physiognomic cues, such as voice, a particular item of clothing, a scar, or gait. Usually patients do not recognize friends, acquaintances, and famous people. In the most severe cases patients cannot even recognize their own face in the mirror. However, perceptual categorization of the stimulus is preserved (patients know that a face is a face) as well as the ability to differentiate faces by gender, race, age, and emotional expressions.

p0285 Psychophysical and neuropsychological studies on face recognition abilities in healthy and brain-damaged patients have revealed that familiar and unfamiliar face processing follows dedicated routes in the left and right hemisphere, respectively. This distinction led the neuropsychologist A.L. Benton to differentiate between two independent face processing deficits produced by brain damage: apperceptive prosopagnosia, which refers to a defective perceptual processing of face information and is brought out by unfamiliar face tasks; and associative prosopagnosia, which involves an additional mnestic component and is elicited by familiar face tasks. ${ }^{38}$

\section{Apperceptive and Associative Prosopagnosia}

According to the model of visual recognition pro- p0290 posed by Bruce and Young, the identification of a face is the final stage in a sequence of operations made by distinct, hierarchically organized, information processing modules distributed along the occipitotemporal ventral pathway of the brain. ${ }^{39}$ At the earlier stages, perceptual face processing results in the construction of an objectcentered, tridimensional structural description of the face. If this processing level is damaged, patients are unable to recognize familiar faces and to match different pictures of unfamiliar faces (apperceptive prosopagnosia). At later processing stages, the structural description of the face activates an abstract representation of it stored in recognition units responsible for the feeling of familiarity. Then, the information gains access to the semantic memory representation (identity nodes) containing the knowledge related to that particular known face. From the identity nodes, information finally accesses the modules containing the person's name. The anatomical-functional independence of the names module is confirmed by the existence of anomia for proper names following left brain damage (a rare occurrence) and by the frequent inability experienced by non-prosopagnosic people to retrieve the name of an otherwise well known person ("name on the tip of the tongue" phenomenon).

Associative prosopagnosia is normally consequent p0295 upon an impairment at the level of either recognition units or identity nodes, or both: patients are able to match unfamiliar faces, but fail to recognize familiar faces. Knowledge of the people to whom the faces belong can be accessed through other sensory modalities (e.g. sound) and is intact.

\section{Unconscious Face Recognition}

This phenomenon is based on the fact that some p0300 prosopagnosic patients may show, in indirect tasks, normal responses to famous faces, thus presenting with some degree of implicit processing of the unrecognized stimuli. This is particularly evident in psychophysiological and neurophysiological measures, such as skin conductance, where prosopagnosic patients may show an increase in electrodermal activity when presented with familiar faces, but not with unfamiliar faces (like healthy subjects). Perhaps the most convincing interpretation of this phenomenon is that conscious recognition requires a higher activation threshold from the visual input than implicit recognition. According to this view, if the lesion producing prosopagnosia completely impairs the function of recognition units, both implicit and explicit recognition will be impossible. If the impairment is only partial, instead, the output from recognition units will be sufficient for unconscious recognition but insufficient for overt recognition (for reviews on agnosia see references ${ }^{40-42}$ ). 
s0195

\section{DISORDERS OF SPATIAL REPRESENTATION: UNILATERAL NEGLECT}

p0305 Unilateral neglect (UN) is a neurological syndrome first described around the turn of the twentieth century. Its core symptom is the loss of conscious awareness for the left side of the perceptual and mental space.

\section{s0200 Clinical Manifestations}

p0310 UN patients behave "... as if they were no longer able to perceive and conceive the existence of the left side of somatic and extrasomatic space". ${ }^{43}$ For example, in the acute phase after stroke, more frequently affecting the right hemisphere, patients often show a more or less complete deviation of the eyes and head towards the right space, fail to respond to left-side visual and auditory stimulation, tend to underuse their left upper and lower limbs (in the absence of hemiplegia or severe motor impairment), do not explore the left side of their body or environment, and may forget to dress the left side of their body or to wash the left side of their face. UN patients are often unaware of their symptoms (anosognosia).

p0315 UN may be diagnosed by means of simple bedside paper-and-pencil tests. When asked to bisect a line segment, UN patients misbisect it to the right of the objective midpoint; when asked to cross out line segments printed on a sheet of paper (Albert's test) they omit to cross out a number of left-side segments; in drawing or recalling from memory simple objects (e.g. a daisy or a clock face) they omit, misarrange, or distort left-side details (Fig. 32.1).

p0320 Neglect symptoms may also occur outside the visual domain, in the haptic (somatic and extrasomatic) space (e.g. blindfolded patients asked to touch their left hand or shoulder with their right hand may fail to do it, or asked to collect objects spread over a table may not find them), the auditory space (patients asked to locate sound sources may mislocate them to the right side of space), and the representational space (patients asked to form the mental image of a familiar view - a town square, a room, a map of a country - and to describe its details from two opposite vantage points may omit left-side details of the mental images).

\section{Clinicoanatomical Correlations}

$\mathrm{UN}$ is more frequent following lesions to the right p0325 hemisphere, with an incidence varying from 30 to $43 \%$ after right hemisphere stroke. ${ }^{43,44} \mathrm{UN}$ may be localized within a hemisphere, and has been associated with both cortical and subcortical lesions. Brain areas whose lesion is most frequently associated with UN are the temporal, parietal, frontal, and occipital lobes, the basal ganglia, and the thalamus. ${ }^{44}$

\section{Course of Unilateral Neglect}

The majority of the symptoms of neglect recede more p0330 or less completely following the acute phase of the disease. However, depending on the severity of neglect, some symptoms may persist for weeks or years. The mechanisms sustaining the functional restoration of neglect symptoms are not yet fully understood and include plastic processes arising in surviving circuits in the damaged hemisphere as well as in structures of the undamaged hemisphere.

\section{Interpretation of the Syndrome}

Since the 1980s, several interpretations of UN have p0335 been proposed, which differ according to the processing level at which the causal mechanisms of the syndrome are thought to operate: the sensory, attentional, and representational level.

\section{Sensory Interpretations}

This group of interpretations considers the causal p0340 mechanisms of UN as relatively "peripheral": contralesional (left) sensory information would go undetected owing to (1) a sort of global extinction phenomenon (stimuli addressed to the damaged hemisphere are more or less correctly perceived if presented in isolation, but are suppressed if given in association with
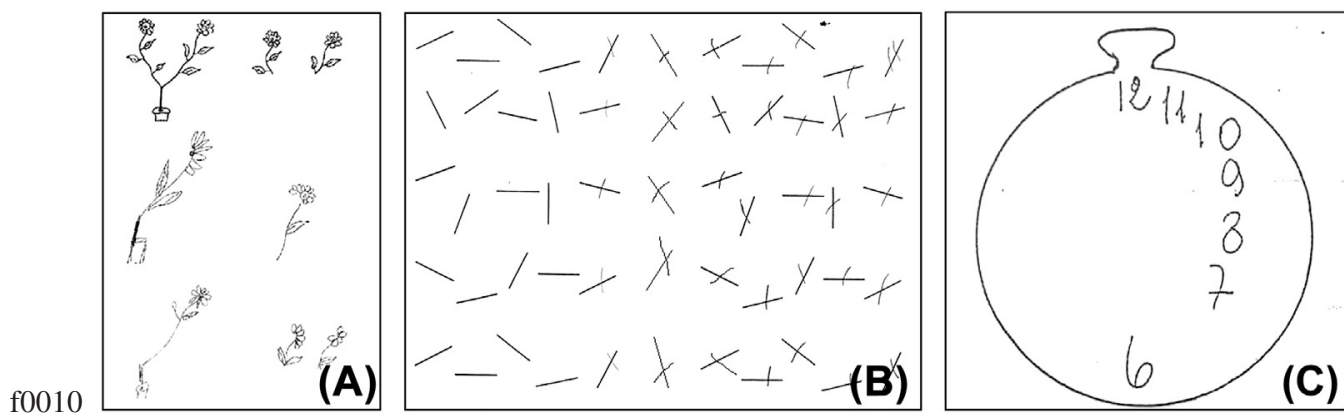

FIGURE $32.1 \quad$ Pencil-and-paper tests in unilateral neglect (UN). (A) Copies of daisies from a model (upper part of the figure) by two patients with UN; (B) Albert's cancellation task: note that the patient has omitted to cancel the majority of left-side segments; $(\mathrm{C})$ drawing from memory of a clock face, by a patient with UN. 
stimuli presented to the undamaged hemisphere); (2) impairment of eye movements towards the left space; or (3) impairment of orienting reflex towards left stimuli. These interpretations refer to impaired processing of stimuli external to the CNS and lost most of their validity after it was ascertained that UN can be found at the level of mental imagery. ${ }^{43}$

\section{s0225 Attentional Interpretations}

p0345 According to these interpretations, lesions to the right hemisphere impair the ability of UN patients to efficaciously orient attention leftwards, with the consequence of becoming unaware of contralesional sensory events. The model that is often used to interpret UN symptoms was proposed by Kinsbourne. ${ }^{45}$ This model posits the existence of two antagonist attentional vectors, each depending on one hemisphere and directing attention contralaterally. A further assumption of the model is that the left hemisphere vector normally predominates over the right hemisphere one, so that the right side of space is attentionally privileged even in healthy people. This model predicts that damage to one hemisphere would impair the corresponding attentional vector and therefore cause a pathological imbalance of attention towards the ipsilesional hemispace. If the left hemisphere is lesioned, the imbalance is less pronounced, and neglect unapparent, because it releases the weaker right hemisphere vector; by contrast, if the right hemisphere is lesioned, the predominant left hemisphere vector is released, biasing attention more strongly rightwards and causing neglect for the left side of space. According to this model, the attentional impairment caused by the lesion should be distributed along the left-right dimension of space, with a continuous gradient ranging from maximum to minimum severity in the left and right extremes spatial sectors, respectively. Behavioral and neurophysiological data confirm this prediction. In this respect, it is worth noting that single-neuron electrophysiology showed that in the postarcuate cortex of the monkey (the lesion of which gives rise to contralateral neglect) $29 \%$ of neurons have exclusively contralateral, $3 \%$ exclusively ipsilateral, and $68 \%$ bilateral receptive fields. ${ }^{46}$ Such a distribution of space-coding neurons is compatible with the gradient of the attentional bias along the left-right dimension of space described for UN patients.

\section{s0230 Representational Interpretations}

p0350 As already mentioned, UN patients asked to remember and describe a familiar view (a town square, a map of their country, or the layout of their home) from a definite vantage point may neglect contralesional details, in the absence of long-term spatial memory deficits. Such pioneering clinical observations, first reported by Edoardo Bisiach and co-workers, ${ }^{47}$ led them to conclude that
$\mathrm{UN}$ is also a deficit of the endogenous mental representation of the contralesional extent of perceptual space. Representational accounts of UN do not entail that attentional interpretations are inadequate; they imply that the concept of attention should be extended to comprise "... the processes of generation and transformation of mental representations" and "... it would therefore refer to the dynamics of representational processes themselves, rather than to separate processes". ${ }^{43}$ Bisiach and colleagues ${ }^{48,49}$ updated the representational explanation in order to explain an apparently paradoxical behavior shown by some neglect patients. When asked to extend a segment towards the left in order to double its original length, some patients overextend it, so as to create a left half-segment much longer than the right halfsegment. This behavior cannot be accounted for by sensory, attentional, and representational interpretations, all predicting absence of or minimal contralesionally directed behavior. Bisiach and co-workers proposed that "This phenomenon may be functionally interpreted as a left-right pathological anisometry of the medium in which within- and between-objects spatial relations are episodically represented. As a consequence of right brain damage, this medium becomes relatively more and more relaxed toward the contralesional and more and more compact toward the ipsilesional side". ${ }^{49}$ Accordingly, any object confronting that scale would shrink on the contralesional side and stretch out on the ipsilesional side, so that UN patients erroneously displace rightwards the subjective midpoint of line segments to be bisected; similarly, when asked to duplicate leftwards a half-segment located ipsilesionally, they will overextend it leftwards in order to subjectively perceive it as identical to the right half-segment (Fig. 32.2).

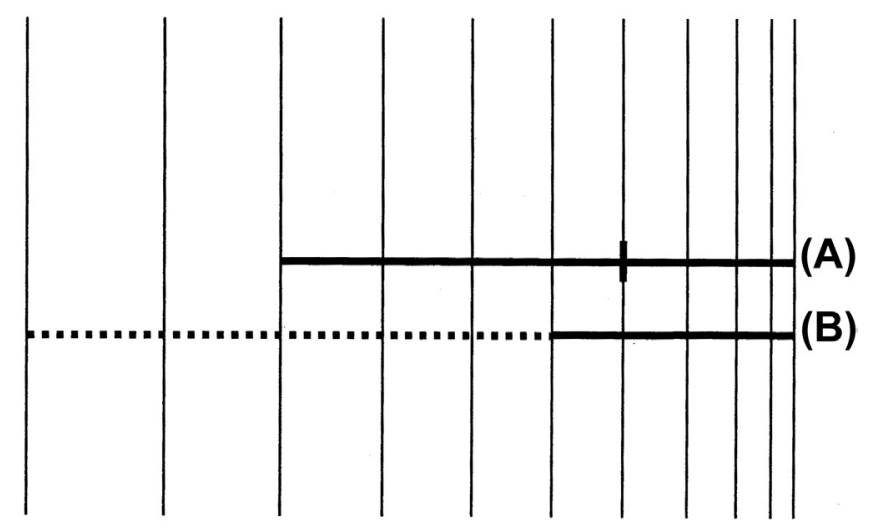

FIGURE 32.2 Progressive leftward relaxation of the medium for space representation in unilateral neglect (UN). (A) Horizonta extension of the left and right sides of a line segment are underestimated and overestimated, respectively, leading UN patients to misplace rightwards the subjective middle of the segment; (B) similarly, they overextend a segment leftwards to double its original length. 


\section{s0235 Implications for Cognitive Science}

p0355 Data collected from UN patients have important theoretical implications for cognitive processes in general and for the understanding of the structure of mental representation in particular. It has long been debated whether the structure of mental contents is symbolic (linguistic) or analogic. The neuropsychological evidence from UN strongly supports the notion of an analogue structure of spatial cognition, similar to the topological relationship existing between external space and the surface of the retina and of the striate visual cortex: "... analogue relationships, such as those existing between perceived objects (and events) and the perceiving system, may also exist between those objects (and events) and the brain mechanisms capable of representing them even in their absence. ... In the same way as lesion of part of the calcarine cortex gives rise to a sensory scotoma, so spatially circumscribed lesions of critical brain structures may give rise to topologically correspondent representational disorders". 43

p0360 Another issue connected with UN is the "destiny" of neglected information: is it completely lost or is it processed at some level? A large body of neuropsychological evidence suggests that neglected information is processed up to a semantic level (see "Neglect: A Model for Spatial Awareness", below).

p0365 A final general remark concerns cognitive models of brain functions. In order to be considered fully reliable in their explanatory power, they need to be grounded on a solid neurobiological basis. This is also true for the representational model of UN, which is still lacking its neurobiological counterpart. Future research should seek an effective explanation of the phenomena of contralesional misrepresentation in the changes that are likely to be induced by unilateral brain lesions in the receptive fields of neurons involved in coding spatial relationships. Unilateral lesions may affect not only the metrics according to which spatial relations are processed in the brain, but also the degree to which, and the way in which, contents organized within a certain area of the pathologically uneven medium reach conscious processing levels. The study of changes in the responsiveness of single space-coding neurons following selective brain lesions may, therefore, shed light on the links between space representation and consciousness.

\section{CONSCIOUS AWARENESS}

p0370 Understanding the relationship between neural activity and subjective conscious experience is one of the most fascinating and challenging goals of modern neuroscience. In this section, this topic will be explored, starting from neuropsychological disorders in which different aspects of conscious awareness can be selectively impaired. In particular, the text will focus on three syndromes, namely blindsight, neglect, and anosognosia for hemiplegia (AHP), that can be used as models for the study of visual, spatial, and motor awareness, respectively. Finally, a modular model of conscious awareness will be proposed.

\section{Blindsight: A Model for Visual Awareness}

Awareness of a stimulus is usually defined as the sub- p0375 jective experience of the existence of a stimulus and the related recognition of its physical and semantic properties. Conscious identification of a visual stimulus can be reported verbally or can be inferred by the observer's overt behavior in response to stimulus presentation. Indeed, if an observer is aware of the presented stimuli, he or she can act upon them. A crucial issue in cognitive neuropsychology has been whether there is the possibility of stimulus elaboration without conscious awareness of it. A convincing example of this possibility is the blindsight syndrome. ${ }^{50,51}$ The phenomenology of blindsight has two principal features. The first is the loss of visual awareness associated with damage to the primary visual cortex (V1; BA17). The second is the residual capacity of blind patients to use visual signals to guide behavioral responses. Pöppel and colleagues' pioneering study showed that these patients, when requested to look at the "unseen" targets, are able to direct their eyes towards these stimuli. ${ }^{52}$ Weiskrantz and colleagues further investigated this phenomenon, showing that blindsight patients, although completely unaware of the presence of a visual stimulus in the blind field, are nevertheless able to point to it correctly and "guess" its orientation..$^{50}$ Since then, many studies have been conducted in many different laboratories around the world, which demonstrated residual processing of different attributes of the unseen stimuli by blindsight patients. For instance, Tamietto and de Gelder explored emotion detection in blindsight. ${ }^{53}$ They demonstrated that patients could correctly guess the emotion expressed by faces presented in their blind field. Therefore, blindsight studies have shown that many stimulus attributes can be processed in the absence of conscious vision.

One possible explanation of this paradoxical phenom- p0380 enon is that even though a large part of the visual cortex is damaged, tiny islands of healthy tissue are spared. The visual processing carried out in this spared tissue is not enough to provide conscious perception, but nevertheless is sufficient to sustain blindsight. In this view, conscious awareness would be a matter of threshold; that is, when, after damage to the brain, the cortical activity is too low and does not reach a sufficient level for conscious activation of sensory input, visual awareness is lost. However, at least in some patients, it has been demonstrated that when no residual island of healthy tissue 
remains, as in surgical resection of V1 or in hemispherectomy, blindsight can still be present. ${ }^{54}$ An alternative hypothesis is that V1 activity has a direct role in generating visual awareness. If so, consciousness would not be a matter of threshold but would depend on the integrity of a specific brain circuit, while the possibility of blindsight would depend on the integrity of a different circuit. In this respect it is important to refer to the complex neuroanatomy of vision. First of all, it must be kept in mind that visual information uses many different pathways from the retina to the brain. The primary visual pathway projects from the retina to V1, in the occipital lobe, via an intermediate station in the lateral geniculate nucleus of the thalamus. From V1, visual information reaches the extrastriate cortex along the ventral (occipitotemporal) and the dorsal (occipitoparietal) stream. However, a minority of fibers originating from the retina takes a secondary route to the superior colliculus and the pulvinar. These two subcortical structures are interconnected and also send direct projections to the extrastriate visual cortex, bypassing V1. Considering these anatomical characteristics of the visual pathways, one possibility is that $\mathrm{V} 1$ is necessary for conscious recognition of visual stimuli. If it is damaged, visual consciousness is prevented. However, the integrity of alternative pathways, such as the retinal-collicular-extrastriate cortex pathway, can allow some visual processing without awareness, as in blindsight patients. Many studies have demonstrated the importance of the collicular nucleus for unconscious vision. Other studies have shown that V1 is necessary, but not sufficient, for full visual awareness. Indeed, other brain regions must be activated (such as prefrontal area 46) to gain full consciousness of visually presented stimuli.

\section{s0250 Neglect: A Model for Spatial Awareness}

p0385 A disorder that has shed light on spatial awareness is the neglect syndrome. Patients with unilateral spatial neglect, in contrast to blindsight patients, may not have any primary visual impairment. Nonetheless, they fail to respond to stimuli, objects, and even people located on their contralesional side, usually the left hemispace (see "Disorders of Spatial Representation: Unilateral Neglect", above).

p0390 Despite patients' behavior suggesting the opposite, it is possible to show that the neglected stimuli can in some cases be fully processed. Marshall and Halligan reported a case of a woman with a severe visual neglect who explicitly denied any difference between the drawing of an intact house and that of a burning house when the features relevant to the discrimination were on the neglected side. ${ }^{55}$ However, when forced to choose the house she would prefer to live in she consistently indicated the non-burning one, showing an implicit knowledge of the information she was unable to consciously report. Further studies showed that the patient's choice was actually based on high-level visual processing. In Berti and Rizzolatti's study, patients were required to respond as rapidly as possible to target stimuli (pictures of animals or vegetables) presented to the normal field by pressing one of two keys according to the category of the targets. ${ }^{56}$ The influence of priming stimuli, pictures of animals or vegetables, presented to the neglected field on target reaction times was studied. By combining different pairs of primes and targets, three different experimental conditions were obtained. In the first condition, "highly congruent", the target and the prime stimuli belonged to the same category and were physically identical; in the second condition, "congruent", the stimuli represented two elements of the same category but were physically dissimilar; in the third condition, "non-congruent", the stimuli represented one exemplar from each of the two categories of stimuli. The results showed that the responses were facilitated not only in the highly congruent condition, but also in the congruent condition, suggesting that patients with neglect are able to process stimuli presented to the neglected field up to a categorical level of representation even when they deny the presence of the stimulus in the neglected field.

The dual visual streams discussed above may be p0395 used to explain non-conscious perception in neglect patients. As already mentioned, visual information from V1 reaches the extrastriate cortex along the ventral (occipitotemporal) and the dorsal (occipitoparietal) stream. The ventral stream (also known as the "what pathway") travels to the temporal lobe and is involved with object identification and recognition. The dorsal stream ("where pathway") terminates in the parietal lobe and is involved with processing the object's spatial location relevant to the viewer. ${ }^{57}$ Because the neglect syndrome is usually associated with parietal lesions, which spare occipitotemporal areas, the demonstration that neglect patients can process visual shapes presented to the neglected side (up to a semantic processing level), although counterintuitive psychologically, is not very surprising. Indeed, the capacity of shape analysis and categorization shown by neglect patients is exactly what one would expect from the functional-anatomical properties of the intact ventral (occipitotemporal) visual pathway. The really surprising aspect of Berti and Rizzolatti's findings is that, despite the presence of semantic elaboration, patients appeared unaware of the stimuli presented in the affected hemispace. ${ }^{56}$ The authors proposed that the encoding of space is a necessary prerequisite for conscious perception. If spatial encoding is prevented or impaired, as it is in neglect, the presence of the stimulus does not reach the conscious level. 
s0255 Anosognosia for Hemiplegia: A Model for Motor Awareness

p0400 Anosognosia for hemiplegia (AHP) is a clinical condition in which movement awareness is dramatically altered. The phenomenon was named anosognosia (from the Greek for "lack of knowledge for the illness") in 1914, by the French neurologist Joseph Babinski. ${ }^{58}$ AHP is usually observed in patients with right-brain damage, who obstinately deny that there is something wrong with their contralesional limbs, despite the presence of severe left paralysis (for a review see Pia et al. ${ }^{59}$ ). If asked to produce an action with the paralyzed limb, some patients appear convinced that they are actually performing it, even though sensory and visual evidence from the affected motionless side should indicate that no movement has been performed.

p0405 Several authors have proposed that AHP may be conceptualized as a selective disorder of motor cognition, ${ }^{60-62}$ on the basis of computational models of motor production and motor control ${ }^{63-65}$ (Fig. 32.3). These models posit that, in the presence of a normal intentional attitude, once the appropriate motor commands have been selected and sent to the muscles for the execution of the desired movement, a prediction of the sensory consequences of the movement is formed and will be successively compared with the feedback associated with the actual execution of the intended movement. According to Blakemore and colleagues, ${ }^{64}$ this prediction, based on the efference copy of the programmed motor act, constitutes the signal upon which motor awareness is constructed. A first consequence of the above-mentioned hypothesis is that if consciousness of a motor act precedes the sensory feedbacks related to a specific movement, ${ }^{67}$ than one should expect to observe motor awareness for a certain movement even in the absence of any observable motor event.
This is exactly what happens in hemiplegic patients p0410 affected by AHP, who seem to be a perfect model to verify the relationship between motor awareness and motor intention. It has been proposed that the denial behavior in AHP patients may be due to direct damage to the comparator system, localized in the premotor (BA6) and insular area. ${ }^{62,68}$ This may impair the motor monitoring process, preventing patients from distinguishing between movement and no-movement states. However, the evident feeling of movement that AHP patients (erroneously) report experiencing may arise from intact motor intentionality, due to normal activity in other areas (mostly involving the parietofrontal circuit) that implement intention-programming related processing, which are usually spared in AHP patients. ${ }^{62}$ Therefore, although AHP patients may not be able to monitor the mismatch between motor prediction and actual execution, because of damage to the comparator, they may still be able to program movements and form predictions, with the consequence of constructing an illusory, but neurologically grounded, motor awareness.

This hypothesis has been confirmed by studies ${ }^{69,70}$ p0415 showing that the subjective experience of movement reported by AHP patients has objective consequences on their motor behavior. Using bimanual motor tasks, in which AHP patients were asked to simultaneously perform movements with both hands, the authors found that the movements of the intact hand were influenced by the intended but not the executed movements of the paralyzed hand. This "influence" produces both spatial $^{69}$ and temporal ${ }^{70}$ coupling effects, comparable to those found in healthy subjects actually performing bimanual tasks. These findings in AHP patients clearly show that motor awareness can be constructed even in the absence of movement execution, solely on the basis of normal intentional processes.

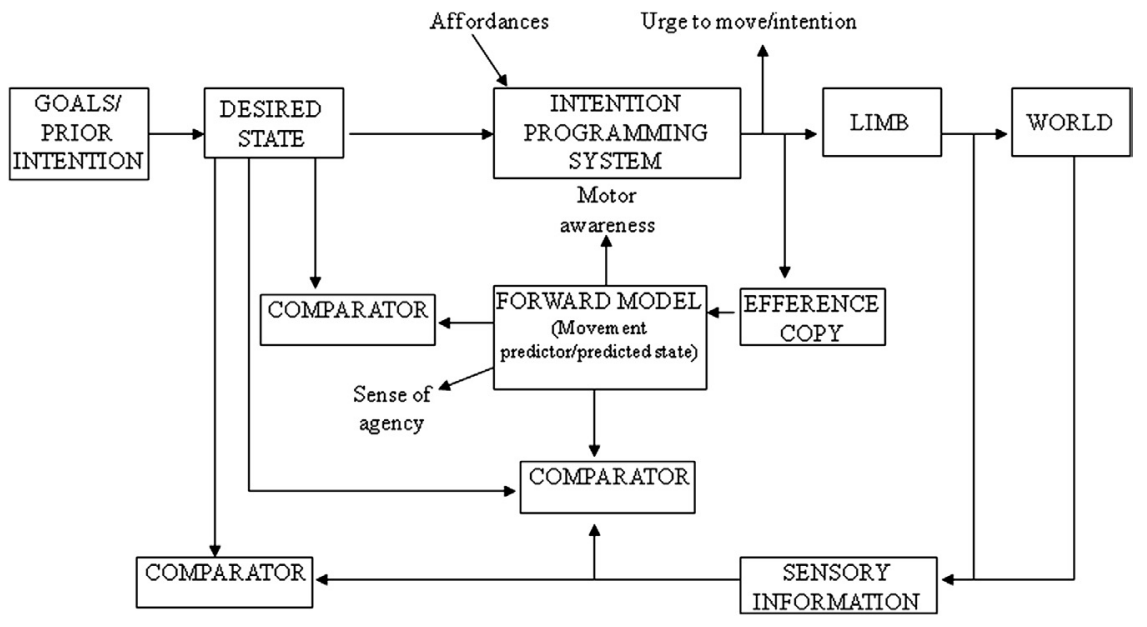

FIGURE 32.3 Anosognosia for hemiplegia. Modified version of the feed-forward model of action generation. 65,66 


\section{s0260 A Modular Model for Conscious Awareness}

p0420 The subjective experience that people have of themselves is reported to be, in normal conditions, a feeling of unity. The "illusion" of unity of the self, assumed by common-sense theories of consciousness, is evident in the normal experience of correspondence between the actual presence or absence of a stimulus and the presence or absence of a subjective experience of it. An alternative view would suggest that consciousness and self-consciousness do not have a unitary, monolithic structure, but instead have a composite nature, subserved by the activity of different brain mechanisms distributed in specialized brain areas. Such a view would predict that focal brain damage should not cause a generalized impairment of conscious experience or conscious self-monitoring, but should instead result in domain-specific disorders of awareness. ${ }^{66}$ The discovery of selective disorders of conscious awareness (such as blindsight, neglect, and AHP) has provided evidence for a composite nature of conscious processes, as opposed to a unitary one. Although the construction of fully conscious states may need the co-occurrence of activity in different parts of the brain, initial modular activation in dedicated brain structures seems to be necessary for domain-specific awareness.

\section{FUTURE DIRECTIONS}

p0425 Throughout this chapter the authors have raised some of the many questions that remain to be answered as researchers drive to achieve a more complete understanding of the multitude of disorders of higher cortical function. To do so will require the combined effort of individuals with expertise in histology, electrophysiology, imaging, and behavioral analysis working both in animal models and in clinical populations. The hope is that some of the readers of this chapter will take up this critical challenge.

\section{s0270 References}

1. Shallice T. From Neuropsychology to Mental Structure. Cambridge, MA: Cambridge University Press; 1988.

2. Broca P. Perte de la parole, ramollissement chronique et destruction partielle du lobe antérieur gauche. Bulletin de la Société d'Anthropologie. 1861;2:235-238.

3. Smith Churchland P. Neurophilosophy. Cambridge, MA: MIT Press; 1986.

4. Lichtheim L. On aphasia. Brain. 1885;7:433-484.

5. Damasio AR. Aphasia. N Engl J Med. 1992;326:531-539.

6. Wernicke C. Der aphasische Symptomencomplex. Breslau: Max Cohn \& Wiegert; 1874.

7. Plaza M, Gatignol P, Leroy M, Duffau H. Speaking without Broca's area after tumor resection. Neurocase. 2009;15:294-310.
8. Anderson VA, Morse SA, Catroppa C, Haritou F, Rosenfeld JV. Thirty month outcome from early childhood injury: a prospective analysis of neurobehavioral recovery. Brain. 2004;124:2608-2620.

9. Varela FJ, Thompson E, Rosch E. The Embodied Mind. Cognitive Science and Human Experience. Boston, MA: MIT Press; 1991.

10. Garbarini F, Adenzato M. At the root of embodied cognition: cognitive science meets neurophysiology. Brain Cogn. 2004;56:100-106.

11. Aziz Zadeh L, Damasio A. Embodied semantics for actions: findings from functional brain imaging. J Physiol. 2008;102:35-39.

12. Lakoff G, Johnson M. Philosophy in the Flesh: The Embodied Mind and Its Challenge to Western Thought. New York: Basic Books; 1999.

13. Hebb DO. The Organization of Behavior. New York: Wiley; 1949.

14. Lashley KS. Brain Mechanisms and Intelligence: A Quantitative Study of Injuries to the Brain. Chicago, IL: Chicago University Press; 1929

15. Scoville WB, Milner B. Loss of recent memory after bilateral hippocampal lesions. J Neurol Neurosurg Psychiatry. 1957;20:11-21.

16. Squire LR. The legacy of patient HM for neuroscience. Neuron 2009;61:6-9.

17. Corkin S. What's new with the amnesic patient HM? Nat Rev Neurosci. 2002;3:153-160.

18. Squire LR, Wixted JT. The cognitive neuroscience of human memory since HM. Annu Rev Neurosci. 2011;34:259-288.

19. Eichenbaum H. What HM taught us. J $\operatorname{Cog} n$ Neurosci. 2012;25: 14-21.

20. Milner B. Les troubles de la memoire accompagnant des lesions hippocampiques bilaterales. In: Passouant $\mathrm{P}$, ed. Physiologie de l'hippocampe. Paris: Centre National de la Recherche Scientifique; 1962:257-272.

21. Corkin S. Tactually-guided maze learning in man: effects of unilateral cortical excisions and bilateral hippocampal lesions. Neuropsychologia. 1965;3:339-351.

22. Corkin S. Acquisition of a motor skill after bilateral medial temporal lobe excision. Neuropsychologia. 1968;6:225-265.

23. Eichenbaum H, Cohen NJ. From Conditioning to Conscious Recollection: Memory Systems of the Brain. New York: Oxford University Press; 2001.

24. Zola-Morgan S, Squire LR, Amaral DG. Human amnesia and the medial temporal region: enduring memory impairment following a bilateral lesion limited to field CA1 of the hippocampus. J Neurosci. 1986;6:2950-2967.

25. Fama R, Pitel AL, Sullivan EV. Anterograde episodic memory in Korsakoff syndrome. Neuropsychol Rev. 2012;22:93-104.

26. Liepmann H. Apraxie. Ergebn ges Med. 1920;1:516-543.

27. Geschwind N. Disconnexion syndromes in animals and man. Brain. 1965;88:237-294.

28. Rothi LJG, Ochipa C, Heilman KM. A cognitive neuropsychological model of limb praxis. Cogn Neuropsychol. 1991;8(6):443-458.

29. Heilman KM, Rothi LJ. Apraxia. In: Heilman KM, Valenstein E, eds. Clinical Neuropsychology. 3rd ed. New York: Oxford University Press; 1993:141-163.

30. Rizzolatti G, Craighero L. The mirror-neuron system. Annu Review Neurosci. 2004;27:169-192.

31. De Renzi E, Faglioni P. Apraxia. In: Denes G, Pizzamiglio L, eds. Handbook of Clinical and Experimental Neuropsychology. Hove: Psychology Press; 1999:421-440.

32. Goldenberg G. Apraxia and the parietal lobes. Neuropsychologia. 2009:47:1449-1459.

33. Petreska B, Adriani M, Blanke O, Billard AG. Apraxia. A review. Prog Brain Res. 2007;164:61-83.

34. Lissauer H. Ein fall von seelenblindheit nebst einem beitrag zur theorie derselben. Arch fur Psychiatrie. 1890;21:222-270.

35. Riddoch MJ, Humphreys GW, eds. Visual Object Processing: A Cognitive Neuropsychological Approach. Hove: Laurence Erlbaum; 1987.

36. Marr D. Vision: A Computational Investigation into the Human Representation and Processing of Visual Information. San Francisco, CA: Freeman; 1980 
37. Warrington EK. Agnosia: The Impairment of Object Recognition. Amsterdam: Elsevier; 1985.

38. Benton AL. The neuropsychology of facial recognition. Am Psychol. 1980;35:176-186.

39. Bruce V, Young A. Understanding face recognition. Br J Psychol. 1986;77:305-327.

40. Behrmann M, Nishimura M. Agnosias. WIREs Cogn Sci. 2010;1: 203-213.

41. De Renzi E. Agnosia. In: Denes G, Pizzamiglio L, eds. Handbook of Clinical and Experimental Neuropsychology. Hove: Psychology Press; 1999:371-407.

42. Farah MJ. Visual Agnosia. Disorders of Object Recognition and What They Tell Us About Normal Vision. Cambridge, MA: MIT Press; 1990.

43. Bisiach E. Unilateral neglect. In: Denes G, Pizzamiglio L, eds. Handbook of Clinical and Experimental Neuropsychology. Hove: Psychology Press; 1999:479-495.

44. Buxbaum L. On the right (and left) track: twenty years of progress in studying hemispatial neglect. Cogn Neuropsychol. 2006;23: 184-201.

45. Kinsbourne M. Mechanisms of unilateral neglect. In: Jeannerod M, ed. Neurophysiological and Neuropsychological Aspects of Spatial Neglect. Amsterdam: Elsevier; 1987:69-86.

46. Rizzolatti G, Gentilucci M, Matelli M. Selective spatial attention: one center, one circuit or many circuits? In: Posner MI, Marin OSM, eds. Attention and Performance XI. Hillsdale, NJ: Erlbaum; 1985:251-265

47. Bisiach E, Luzzatti C. Unilateral neglect of representational space. Cortex. 1978:14:129-133.

48. Bisiach E, Pizzamiglio L, Nico D, Antonucci G. Beyond unilateral neglect. Brain. 1996;119:851-857.

49. Bisiach E, Ricci R, Neppi-Modona M. Visual awareness and anisometry of space representation in unilateral neglect: a panoramic investigation by means of a line extension task. Consc Cogn. 1998;7:327-355

50. Weiskrantz L, Warrington EK, Sanders MD, Marshall J. Visual capacity in the hemianopic field following a restricted occipital ablation. Brain. 1974;97:709-728.

51. Leopold DA. Primary visual cortex: awareness and blindsight. Annu Rev Neurosci. 2012;35:91-109.

52. Pöppel E, Held R, Frost D. Residual visual function after brain wounds involving the central visual pathways in man. Nature. 1973;243:295-296.

53. Tamietto M, de Gelder B. Neural bases of the non-conscious perception of emotional signals. Nat Rev Neurosci. 2010;11:697-709.
54. Tomaiuolo F, Ptito M, Marzi CA, Paus T, Ptito A. Blindsight in hemispherectomized patients as revealed by spatial summation across the vertical meridian. Brain. 1997;120:795-803

55. Marshall JC, Halligan PW. Blindsight and insight in visuo-spatial neglect. Nature. 1988;336:766-767.

56. Berti A, Rizzolatti G. Visual processing without awareness: evidence from unilateral neglect. I Cogn Neurosci. 1992;4:345-351.

57. Goodale MA, Milner AD. Separate visual pathways for perception and action. Trends Neurosci. 1992;15:20-25.

58. Babinski J. Contribution à l'étude des troubles mentaux dan l'hémiplégie organique cérébrale (anosognosie). Revue Neurologique. 1914;27:845-848.

59. Pia L, Neppi-Mòdona M, Ricci R, Berti A. The anatomy of anosognosia for hemiplegia: a meta-analysis. Cortex. 2004;40:367-377.

60. Gold G, Adair JC, Jacobs DH, Heilman KM. Anosognosia for hemiplegia: an electrophysiologic investigation of the feed-forward hypothesis. Neurology. 1994;44:1804-1808.

61. Frith CD, Blakemore SJ, Wolpert DM. Abnormalities in the awareness and control of action. Philos Trans $R$ Soc Lond B Biol Sci. 2000;355:1771-1788.

62. Berti A, Bottini G, Gandola M, et al. Shared cortical anatomy for motor awareness and motor control. Science. 2005;309:488-491.

63. Wolpert DM, Ghahramani Z, Jordan MI. An internal model for sensorimotor integration. Science. 1995;269:1880-1882.

64. Blakemore SJ, Wolpert DM, Frith CD. Abnormalities in the awareness of action. Trends Cogn Sci. 2002;6:237-242.

65. Haggard P. Conscious intention and motor cognition. Trends Cogn Sci. 2005:9:290-295.

66. Spinazzola L, Pia L, Folegatti A, Marchetti C, Berti A. Modular structure of awareness for sensorimotor disorders: evidence from anosognosia for hemiplegia and anosognosia for hemianaesthesia. Neuropsychologia. 2008;46:915-926.

67. Libet B, Gleason CA, Wright EW, Pearl DK. Time of conscious intention to act in relation to onset of cerebral activity (readinesspotential). The unconscious initiation of a freely voluntary act. Brain 1983;106:623-642.

68. Karnath HO, Baier B, Nagle T. Awareness of the functioning of one's own limbs mediated by the insular cortex? I Neurosci. 2005;25:7134-7138

69. Garbarini F, Rabuffetti M, Piedimonte A, et al. "Moving" a paralysed hand: bimanual coupling effect in patients with anosognosia for hemiplegia. Brain. 2012;135:1486-1497.

70. Pia L, Spinazzola L, Rabuffetti M, et al. Temporal coupling due to illusory movements in bimanual actions: evidence from anosognosia for hemiplegia. Cortex. 2013;49(6):1694-1703. 


\title{
ZIGMOND: 32
}

\section{Non-Print Items}

\begin{abstract}
A brief overview of the most important cognitive alterations a consequence of focal brain damage is presented. The discipline that studies the relationship between brain damage and higher cognitive functions is called neuropsychology and is mainly based on correlations between anatomy and function. Leaving aside the importance of the clinical impact that neuropsychology has on patients' treatment, its main experimental aim is to draw inferences from the pathological conditions to normal functions, on the assumption that if the lesion to a particular brain area or circuit has provoked a specific deficit, then that part of the brain is involved in or is necessary for sustaining the normal function. The main classical neuropsychological syndromes related to perceptual, spatial, linguistic, motor, and memory domain will be discussed, with a final part on syndromes in which conscious awareness is specifically altered.
\end{abstract}

Keywords: agnosia; amnesia; anosognosia for hemiplegia; aphasia; apraxia; blindsight; conscious awareness; embodied semantic; motor control; neglect. 